\title{
UTILIZAÇÃO DE UM DELINEAMENTO COMPOSTO CENTRAL ROTACIONAL (DCCR) PARA A PRODUÇÃO DE BIOSSURFACTANTE POR PSEUDOMONAS AERUGINOSA
}

\author{
P. F. CORRÊA ${ }^{1}$, J. G. M. BRITO ${ }^{1}$, H. S. AMORIM ${ }^{1}$, B.G. FREITAS $^{1}$, R. D. RUFINO ${ }^{1,2}$, \\ J.M. LUNA ${ }^{1,2}$, L.A. SARUBBO ${ }^{1,2}$ \\ ${ }^{1}$ CCT - Universidade Católica de Pernambuco, Centro de Ciências e Tecnologia \\ ${ }^{2}$ Centro de Gestão de Tecnologia e Inovação (CGTI) \\ E-mail para contato: priscilla.correa.pe@gmail.com
}

\begin{abstract}
RESUMO - Os constantes derramamentos de óleos causados por empresas petrolíferas vêm causando grandes impactos no ambiente. Nesse contexto, os biossurfactantes são produzidos por micro-organismos e destacam-se na remoção de petroderivados em ambientes marinhos e terrestres. O objetivo, portanto, foi produzir um biossurfactante pela bactéria Pseudomonas aeruginosa UCP 992, cultivada em $0,5 \%$ de milhocina e 4,0\% de resíduo de óleo vegetal em biorreator de 1,2 L, empregando um Delineamento Composto Central Rotacional (DCCR). As condições de cultivo foram velocidade de rotação, tempo, aeração e tamanho do inóculo, avaliando-se a redução da tensão superficial e o rendimento. Os melhores resultados foram uma aeração de $0,5 \mathrm{vvm}$, com 4,0\% do inóculo, a $250 \mathrm{rpm}$ e durante 84 horas, resultando em uma tensão superficial de $25,868 \mathrm{mN} / \mathrm{m}$ e uma extração de 20,855 g/L de biossurfactante. O DCCR identificou as melhores condições para produção do biossurfactante e apresentou propriedades promissoras da biomolécula para biorremedição
\end{abstract}

\section{Introdução}

A necessidade de remediar áreas contaminadas por óleo levou ao desenvolvimento de novas tecnologias de detoxificação destes contaminantes de forma não convencional, ou seja, sem a utilização de métodos somente químicos ou físicos (Fracchia et al., 2012). O uso de micro-organismos ou produtos microbianos para degradar compostos poluentes, é uma destas novas tecnologias (Muthusamy et al., 2008; Singh et al., 2007), conhecida como biorremediação (Calvo et al., 2009).

A biorremediação pode ser definida como um processo de estimulação de situações naturais de biodegradação para limpeza de derramamentos de óleos e tratamento de ambientes terrestres e aquáticos contaminados com compostos xenobióticos (Mukherjee et al., 2006). 
Um dos problemas associados a biodegradação de compostos hidrofóbicos, aos quais incluem-se os hidrocarbonetos do petróleo é sua ligação às partículas do solo e a pouca solubilidade em água, resultando em baixa biodisponibilidade para os micro-organismos, o que pode retardar ou até mesmo paralisar o processo de degradação (Satpute et al., 2010).

Nesse sentido, a utilização de compostos surfactantes torna-se uma alternativa atrativa na remoção de contaminantes hidrofóbicos gerados pela indústria de petróleo.

Os surfactantes são compostos anfipáticos que se particionam, preferencialmente, na interface entre fases fluidas com diferentes graus de polaridade, apresentando várias aplicações industriais (Burghoff et al., 2012). Os surfactantes possuem estrutura molecular com grupos hidrofílicos e hidrofóbicos que exibem propriedades como adsorção, formação de micelas, formação de macro ou micro emulsões, ação espumante, solubilidade e detergência, todas ligadas à capacidade de redução da tensão superficial por essas moléculas (Torres et al., 2011).

A grande maioria dos surfactantes disponível comercialmente é sintetizada a partir de derivados de petróleo (Marchant et al., 2012a). Entretanto, a necessidade de preservação ambiental e as legislações de controle do ambiente têm levado pesquisadores à procura por produtos naturais como alternativas aos produtos existentes. Nesse contexto, destacam-se os surfactantes de origem microbiológica, produzidos principalmente por bactérias e leveduras (Cortis et al., 2007).

Os biossurfactantes incluem uma grande variedade de estruturas químicas tais como glicolipídeos, lipopeptídeos, complexos proteínas-polissacarídeos, fosfolipídeos, ácidos graxos e lipídeos neutros produzidos por micro-organismos quando cultivados em substratos insolúveis (óleos, resíduos e hidrocarbonetos) e solúveis (carboidratos) (Gautam et al., 2006).

Os primeiros estudos na área de biossurfactantes ocorreram na década de $80 \mathrm{e}$, desde então, as pesquisas permitiram o desenvolvimento e a comercialização de dois produtos, a Surfactina, uma lipoproteína produzida pela bactéria Bacillus subtilis (Al-Bahry et al., 2012) e os Raminolipídeos (Aparna et al., 2012), grupo de glicolipídeos produzidos pela bactéria Pseudomonas aeruginosa e comercializados pela Jeneil Biosurfactants Company (USA). Esses dois biossurfactantes, embora extremamente eficientes, são comercializados a um alto custo em função dos substratos utilizados para suas produções e do nível de pureza exigido para aplicações nas áreas farmacêutica e médica (Abdel-Mawgoud et al., 2010; Barros et al., 2007; Seydlová et al., 2008). Os biossurfactantes de Pseudomonas aeroginosas são capazes de reduzir valores de tensão superficial para $29 \mathrm{mN} / \mathrm{m}$, podendo ser produzidos a partir de vários substratos solúveis e insolúveis (Rufino et al., 2010)

Uma das alternativas para reduzir os custos relacionados aos biossurfactantes consiste na substituição dos substratos comumente utilizados por matérias-primas de baixo custo, como os resíduos industriais (Maneerat et al., 2005). Nesse contexto, as indústrias de petróleo e petroquímica destacam-se como os maiores campos de aplicação dos biossurfactantes, uma vez que utilizam esses compostos em sua forma bruta, ou seja, sem necessidade de purificação, o que representa uma acentuada redução nos custos de aplicação de um agente biotecnológico.

Nos últimos anos, os estudos voltados para a produção de biossurfactantes têm se intensificado em função das características desses compostos como biodegradabilidade, baixa toxicidade, especificidade e estabilidade sob condições ambientais extremas de temperatura, pH e salinidade (Felse et al., 2006; Mukherjee et al., 2006).

Nesse sentido, pretende-se utilizar o Delineamento Composto Central Rotacional (DCCR) como detector da melhor condição para a produção de biossurfactante por Pseudomonas aeruginosa na biorremedição. 


\section{MATERIAL E MÉTODOS}

\subsection{Micro-organismo}

A bactéria $P$. aeruginosa (UCP 0992), depositada no Banco de Culturas do Núcleo de Pesquisas em Ciências Ambientais (NPCIAMB) da UNICAP foi utilizada como microorganismo produtor do biossurfactante. As culturas foram repicadas a cada 30 dias e mantidas em tubos de ensaio inclinados com o meio solido Ágar Nutriente (AN) sob refrigeração a $5^{\circ} \mathrm{C}$.

\subsection{Meios de manutenção e crescimento do inóculo de produção do biossurfactante}

Para a manutenção da bactéria foi utilizado o meio Ágar Nutriente com a seguinte composição: extrato de carne $(5,0 \mathrm{~g})$, peptona $(10.0 \mathrm{~g}), \mathrm{NaCl}(5,0 \mathrm{~g})$, ágar $(5,0 \mathrm{~g})$ e água destilada $(1000,0 \mathrm{ml})$. Os constituintes foram solubilizados e esterilizados em autoclave a $121^{\circ} \mathrm{C}$ por 20 minutos. Para crescimento do inóculo foi utilizado o meio Caldo Nutritivo $(\mathrm{CN})$ que possui a mesma composição do $\mathrm{AN}$ sem a presença do ágar. $\mathrm{O}$ meio de produção foi composto com água destilada contendo $0,5 \%$ de milhocina e $4 \%$ de resíduo de borra de refinaria de óleo vegetal (Farias et al., 2007).

\subsection{Preparação do inóculo}

Culturas jovens da bactéria obtidas após 24 horas de cultivo em meio AN foram transferidas para um Erlenmeyer contendo $50 \mathrm{~mL}$ de Caldo Nutritivo que foi mantido sob agitação orbital de $200 \mathrm{rpm}$ durante um período de 14 horas a $28^{\circ} \mathrm{C}$ para obtenção de uma D.O. de 0,7 (correspondente a um inóculo de $10^{7}$ U.F.C./mL) a $600 \mathrm{~nm}$. Esta leitura foi utilizada como inóculo na concentração de $1 \%$ (v/v).

\subsection{Produção do biossurfactante utilizando planejamento fatorial}

As fermentações para produção do biossurfactante foram realizadas em biorreator de 1,2L. O meio de produção foi submetido à variação das condições de cultivo (velocidade de rotação, tempo de cultivo, aeração e tamanho do inóculo) de acordo com um Delineamento Composto Central Rotacional (DCCR) apresentado nas tabelas 01 e 02, à temperatura de $28^{\circ} \mathrm{C}$. Ao fim do cultivo, amostras foram centrifugadas e filtradas para determinação da tensão superficial e extração, como parâmetros utilizados para o critério de seleção da melhor condição de produção.

Tabela 1 - Valores das variáveis independentes nos níveis $-2,-1,+1$ e +2 e no ponto central

\begin{tabular}{ccccc}
\hline Nível & Agitação (rpm) & Aeração (vvm) & Tempo de cultivo (horas) & Tamanho do inóculo (\%) \\
\hline-2 & 175 & 0 & 72 & 1 \\
-1 & 200 & 0,5 & 84 & 2 \\
0 & 225 & 1,0 & 96 & 3 \\
+1 & 250 & 1,5 & 108 & 4 \\
+2 & 275 & 2,0 & 120 & 5 \\
\hline
\end{tabular}

Tabela 2 - Matriz do Delineamento Composto Central Rotacional (DCCR)

\begin{tabular}{ccccc}
\hline Ensaios & Agitação & Aeração & Tempo de cultivo & Tamanho do inóculo \\
\hline $\mathbf{1}$ & -1 & -1 & -1 & -1 \\
$\mathbf{2}$ & +1 & -1 & -1 & -1
\end{tabular}




\begin{tabular}{ccccc}
$\mathbf{3}$ & -1 & +1 & -1 & -1 \\
$\mathbf{4}$ & +1 & +1 & -1 & -1 \\
$\mathbf{5}$ & -1 & -1 & +1 & -1 \\
$\mathbf{6}$ & +1 & -1 & +1 & -1 \\
$\mathbf{7}$ & -1 & +1 & +1 & -1 \\
$\mathbf{8}$ & +1 & +1 & +1 & -1 \\
$\mathbf{9}$ & -1 & -1 & -1 & +1 \\
$\mathbf{1 0}$ & +1 & -1 & -1 & +1 \\
$\mathbf{1 1}$ & -1 & +1 & -1 & +1 \\
$\mathbf{1 2}$ & +1 & +1 & -1 & +1 \\
$\mathbf{1 3}$ & -1 & -1 & +1 & +1 \\
$\mathbf{1 4}$ & +1 & -1 & +1 & +1 \\
$\mathbf{1 5}$ & -1 & +1 & +1 & +1 \\
$\mathbf{1 6}$ & +1 & +1 & +1 & +1 \\
$\mathbf{1 7}$ & -2 & 0 & 0 & 0 \\
$\mathbf{1 8}$ & +2 & 0 & 0 & 0 \\
$\mathbf{1 9}$ & 0 & -2 & 0 & 0 \\
$\mathbf{2 0}$ & 0 & +2 & 0 & 0 \\
$\mathbf{2 1}$ & 0 & 0 & -2 & 0 \\
$\mathbf{2 2}$ & 0 & 0 & +2 & 0 \\
$\mathbf{2 3}$ & 0 & 0 & 0 & -2 \\
$\mathbf{2 4}$ & 0 & 0 & 0 & +2 \\
$\mathbf{2 5}$ & 0 & 0 & 0 & 0 \\
$\mathbf{2 6}$ & 0 & 0 & 0 & 0 \\
\hline
\end{tabular}

\subsection{Determinação da tensão superficial}

A tensão superficial foi medida no líquido metabólico livre de células em tensiômetro KSV Sigma 700 (Finland) utilizando-se o anel de NUOY.

\subsection{Isolamento do biossurfactante}

O biossurfactante produzido foi isolado de acordo com a Costa et al., (2005).

\section{RESULTADOS E DISCUSSÃO}

Surfactantes são compostos químicos sintéticos derivados do petróleo que possuem porções hidrofílicas e hidrofóbicas (Rufino et al., 2014). Já os biossurfactantes são compostos químicos derivados das características de micro-organismos e são menos agressivos ao meio ambiente (Luna et al., 2013).

Apesar da diversidade de composição química e propriedades, algumas características são comuns à maioria dos biossurfactantes, apresentando vantagens sobre surfactantes convencionais, como: altas atividades superficiais e interfaciais, tolerância à temperatura $\mathrm{pH}$ e força iônica, biodegradabilidade e ainda baixa toxicidade (Rufino et al., 2014).

A tensão superficial é definida como a entalpia superficial livre, por unidade de área, e é a força que atua sobre a superfície de um líquido que conduz a minimização da área superficial (Luna et al., 2011). Desta forma as biossurfactantes podem ser responsáveis pela diminuição da tensão superficial dos líquidos devido à fácil distribuição nas interfaces entre as superfícies com diferentes graus de polaridade, através da formação de micelas (Rufino et al., 2014). 
A tabela 03 apresenta os valores das tensões superficiais dos diferentes meios utilizados para a produção do biossurfactante por Pseudomonas aeruginosa (UCP 0992). As medidas foram realizadas no líquido metabólico livre de células para cada condição específica, de acordo com um Delineamento Composto Central Rotacional (DCCR).

Tabela 03- Tensões superficiais e extrações de produção de biossurfactante por Pseudomonas aeruginosa (UCP 0992) utilizando diferentes condições de cultivo

\begin{tabular}{ccc}
\hline Condições & Tensão superficial $(\mathbf{m N} / \mathbf{m})$ & Extração $(\mathbf{g} / \mathbf{L})$ \\
\hline 1 & 27,989 & 3,788 \\
2 & 27,273 & 5,648 \\
3 & 26,226 & 9,847 \\
4 & 28,848 & 9,876 \\
5 & 27,126 & 7,328 \\
6 & 27,555 & 14,424 \\
7 & 26,038 & 9,670 \\
8 & 26,594 & 18,760 \\
9 & 29,546 & 6,090 \\
10 & 25,868 & 20,855 \\
11 & 26,153 & 18,386 \\
12 & 26,366 & 12,50 \\
13 & 26,397 & 20,170 \\
17 & 26,868 & 10,270 \\
19 & 25,949 & 17,523 \\
21 & 28,009 & 4,018 \\
\hline
\end{tabular}

A condição 10 do Delineamento Composto Central Rotacional (DCCR) apresentou melhores resultados em relação às demais condições avaliadas. A condição selecionada apresentou as seguintes condições: inóculo de $4 \%$, cultivo durante 84 horas, aeração de 0,5 vvm e uma agitação de $250 \mathrm{rpm}$. A tensão superficial apresentou um valor de $25,868 \mathrm{mN} / \mathrm{m}$ com uma extração de $20,855 \mathrm{~g} / \mathrm{L}$. O maior rendimento obtido na condição aqui especificada pode estar relacionado ao fato de a bactéria $P$. aeruginosa ser um micro-organismo facultativo que pode apresentar crescimento em ambientes com baixa concentração de oxigênio (Silva $e t$ al., 2010). Os demais experimentos apresentaram resultados favoráveis em relação a menores valores de tensão superficial, mas a seleção da melhor condição de cultivo deverá ser realizada após a conclusão de todos os experimentos e a elaboração de superfícies de resposta contendo os dados obtidos.

Os resultados obtidos são comparáveis aos observados por Silva et al. (2010), onde o biossurfactante produzido por $P$. aeruginosa cultivada em meio contendo $3 \%$ de glicerol apresentou uma tensão superficial de $27,6 \mathrm{mN} / \mathrm{m}$ com uma extração de $6,5 \mathrm{~g} / \mathrm{L}$. Silva et. al. (2013), constatou também que a $P$. cepacia cultivada em meio de $2 \%$ de óleo residual de fritura e $3 \%$ de milhocina produziu um biossurfactante que apresentou uma tensão superficial de $26 \mathrm{mN} / \mathrm{m}$ com uma extração de $8 \mathrm{~g} / \mathrm{L}$.

Aparna et al. (2012), observaram que o biossurfactante produzido por Pseudomonas sp 2B cultivada em meio contendo $1 \%$ de melaço apresentou tensão superficial de $30,14 \mathrm{mN} / \mathrm{m}$ com uma extração de 4,97 g/L. Em estudos realizados por Oliveira et al. (2009), o biopolímero produzido por $P$. alcaligenes PCL cultivada em meio suplementado com sais minerais e óleo de palma, apresentou uma redução na tensão superficial do meio para 28 
$\mathrm{mN} / \mathrm{m}$ com uma extração de 2,3 g/L. Em outro estudo Monteiro et al. (2007), constataram que o biossurfactante produzido por $P$. aeruginosa DAUPE 614 cultivada em meio contendo glicerol e nitrato de amônia apresentou tensão superficial de $27,3 \mathrm{mN} / \mathrm{m}$ com uma extração de $3,9 \mathrm{~g} / \mathrm{L}$.

Sendo assim, pode-se observar que a maioria dos trabalhos realizados com a bactéria Pseudomonas apresentam resultados satisfatórios para a produção de biossurfactantes potentes, em relação à redução das tensões superficiais dos meios de cultivo. Mas a extração observada no presente trabalho foi bem superior aos obtidos nos demais estudos. Assim, o biopolímero obtido nas condições aqui especificadas, torna-se um agente promissor para utilização em experimentos futuros de avaliação de suas características emulsionantes e dispersantes, para que dessa forma sejam avaliadas possibilidades de utilização do bioproduto em ambientes contaminados com petróleo e derivados.

\section{CONCLUSÃO}

Os biossurfactantes podem ser utilizados em substituição aos surfactantes sintéticos, pois não agridem o meio ambiente; entretanto, a produção dessas biomoléculas em grande escala ainda é cara. Desta forma, estão sendo realizados estudos que utilizam meios de produção de baixo custos que permitam a produção de biossurfactantes com características muito próximas às dos surfactantes sintéticos.

A Pseudomonas aeruginosa, (UCP 0992) na presença de milhocina e borra de óleo vegetal, produz um biocomposto com a propriedade surfactante tensão superficial ótima para uma biorremediação. As tensões superficiais foram muito próximas e em algumas condições a extração foi excelente; contudo, ainda é necessário fazer mais fermentações para obter a melhor condição com uma tensão superficial baixa e uma alta extração.

\section{REFERÊNCIAS}

ABDEL-MAWGOUD, A M; LÉPINE, F; DÉZIEL, E. Rhamnolipids: diversity of structures, microbial origins and roles. Applied Microbiology And Biotechnology, v. 86, n. 5, p.13231336, 2010.

AL-BAHRY, S.N.; AL-WAHAIBI, Y.M.; ELSHAFIE, A.E.; AL-BEMANI, A.S.; JOSHI, S.J.; AL-MAKHMARI, H.S.; AL-SULAIMANI, H.S. Biosurfactant production by Bacillus subtilis B20 using date molasses and its possible application in enhanced oil recovery. International Biodeterioration and Biodegradation. doi:10.1016/j.ibiod.2012.01.0062012, 2012.

APARNA, A.; SRINIKETHANA, G.; SMITHAB, H. Production and characterization of biosurfactant produced by a novel Pseudomonas sp. 2B. Colloids and Surfaces B: Biointerfaces. doi:10.1016/j.colsurfb.2012.01.043, 2012.

BARROS, F. F. C.; QUADROS, C. P.; MARÓSTICA, M. R.; PASTORE, M. G. Surfactina: propriedades químicas, tecnológicas e funcionais para aplicações em alimentos. Química Nova. v. 30, n. 2, p. 01-14, 2007.

BURGHOFF, B. Foam fractionation applications. Journal of Biotechnology, v. 161, p. 126- 
137, 2012.

CALVO, $\mathrm{C}$ et al. APPLICATION OF BIOEMULSIFIERS IN SOIL BIOREMEDIATION PROCESSES: FUTURE PROSPECTS. Science Of The Total Enivironment, v. 407, n. , p.3634-3640, 2009.

CORTIS, A.; GHEZZEHEI, T. A. On the transport of emulsions in porous media. Journal of Colloid and Interface Science, v. 313, p. 1-4, 2007.

FELSE, P. A.; SHAH, V.; CHAN, J.; RAO, K. J.; GROSS, R. A. Sophorolipid biosynthesis by Candida bombicola from industrial fatty acid residues. Enzyme and Microbial Technology, 2006.

FRACCHIA, L.; CAVALLO, M.; GIOVANNA MARTINOTTI; M., BANAT, I.M. Biosurfactants and bioemulsifiers biomedical and related applications - Present Status and Future Potentials, Biomedical Science, Engineering and Technology, Dhanjoo N. Ghista (Ed.), pp. 325-370, 2012.

GAUTAM, K. K.; TYAGI, V. K. Microbial Surfactants: a review. Journal of Oleo Science, v. 55, p. 155-166, 2006.

LUNA, J.M.; RUFINO, R.D.; SARUBBO, L.A.; CAMPOS-TAKAKI, G.M. Characterisation, surface properties and biological activity of a biosurfactant produced from industrial waste by Candida sphaerica UCP0995 for application in the petroleum industry. Colloids and Surfaces B: Biointerfaces, v. 102, p. 202-209, 2013.

LUNA, J.M; RUFINO, R.D; ALBUQUERQUE, C.D.C; SARUBBO, L.A.; CAMPOSTAKAKI, G.M. Economic Optimized Medium for Tensio-Active Agent Production by Candida sphaerica UCP0995 and Application in the Removal of Hydrophobic Contaminant from Sand. International Journal of Molecular Sciences. v. 12, p. 2463-2476, 2011.

MANEERAT, S. Production of biosurfactants using substrates from renewable-resources. Songklanakarin Journal of Science and Technology, v. 27, p. 675-683, 2005.

MARCHANT, R.; BANAT, I.M. Biosurfactants: a sustainable replacement for chemical surfactants? Biotechnology Letters, v. 34, p. 1597-1605, 2012a

MONTEIRO S. A.; SASSAKI G. L., SOUZA L. M. DE; MEIRA J. A.; ARAUJO J. M. DE; MITCHELL D. A;, RAMOS L. P.; KRIEGER N. Molecular and structural characterization of the biosurfactant produced by Pseudomonas aeruginosa DAUPE 614. Chemistry and Physics of Lipids, V. 147, p. 1-13, 2007.

MUKHERJEE, S.; DAS, P.; SEN, R. Towards commercial production of microbial surfactants. Trends in Biotechnology, v. 24, p. 509-515, 2006.

MUTHUSAMY, K.; GOPALAKRISHNAN, S.; RAVI, T,K.; SIVACHIDAMBARAM, P. Biosurfactants: properties, commercial production and application. Current Science, v. 94, p. 736-747, 2008.

OLIVEIRA, F.J.S; VAZQUEZ L.; DE CAMPOS N.P.; DE FRANÇA F.P. Production of rhamnolipids by a Pseudomonas alcaligenes strain. Process Biochemistry, v. 44, p. 383-389, 2009

RUFINO, R. D.; SARUBBO, L. A.; CAMPOS-TAKAKI G. M. Enhancement of stability of 
biosurfactant produced by Candida lipolytica using industrial residue as substrate. World Journal of Microbiology and Biotechnology, v. 23, p. 729-734, 2007.

RUFINO,R. D. Produção otimizada do Biossurfactante Rufisan por Candida lipolytica e aplicações biotecnológicas. Tese de doutorado - Universidade Federal de Pernambuco. P. 159, 2014.

SATPUTE, S K et al. Biosurfactants, bioemulsifiers and exopolysaccharides from marine microoganisms. Biotechnology Advances, v. 28, n. , p.436-458, 2010.

SEYDLOVÁ, G.; SVOBODOVÁ, J. Review of surfactin chemical properties and the potential biomedical applications, Central European Journal of Medicine, v. 2, p. 123-133, 2008.

SILVA, S.N.R.L., FARIAS, C.B.B., RUFINO, R.D., LUNA, J.M., SARUBBO, L.A. Glycerol as substrate for the production of biosurfactant by Pseudomonas aeruginosa UCP0992. Colloids and Surfaces. B, Biointerfaces. , v.79, p.174 - 183, 2010.

SILVA, S.N.R.L., RUFINO, R.D., LUNA, J.M, FARIAS, C.B.B., SANTOS, V. A., FILHO, H.J.B.L., SARUBBO, L.A. Enhancement of Biosurfactant production from Pseudomonas cepacia CCT6659 Through Optimisation of Nutritional Parameters Using Response Surface Methodology. Revista Tenside Surfactants Detergents. v. 50, n²2, p. 137-142, 2013.

SINGH, A.; VAN HAMME, J.D.; WARD, O. P. Surfactants in microbiology and biotechnology: Part 2. Application aspects. Biotechnology Advances, v. 25, p. 99-121, 2007.

TORRES, L.; MOCTEZUMA A.; AVENDAÑO, J.R.; MUÑOZ, A.; GRACIDA, J. Comparison of bio- and synthetic surfactants for EOR. Journal of Petroleum Science and Engineering, v. 76 p. 6-11, 2011 